\title{
A Review of Process Parameters That Effect to Extrusion on Demand
}

\author{
Paphakorn Pitayachaval ${ }^{1}$, Thanakhan Baothong ${ }^{1}$ \\ ${ }^{1}$ School of Industrial Engineering, Institute of Engineering, Suranaree University of Technology, Thailand
}

\begin{abstract}
An additive Manufacturing (AM) process has been introduced to fabricate three-dimension objects from liquid polymers and powder particles e.g. polymers and metals, this process has been also applied to fabricate threedimension ceramic objects called paste materials which compose of liquid and solid components. To build additive model, the paste material is extruded via pressure force through the nozzle by using ram extrusion, then the model is constructed layer-by-layer on the table to form designed shape. To improve a capability of extrusion process, the Extrusion On Demand (EOD), that refers to the ability of paste extrusion to regulate start and stop, has been developed to improve an accuracy of material deposition. This paper presents a review of process parameters that effect to extrusion on demand such as ram velocity, Dwell time, paste property and extrusion mechanisms.
\end{abstract}

\section{Introduction}

Additive manufacturing (AM) is a technology that fabricate three-dimensional (3D) objects by adding material layer-by-layer directly from CAD (Computer Aided Design) model [1]. Traditional AM technologies have been used to fabricate $3 \mathrm{D}$ objects based on a raw material states that are liquid-based, solid-based, and powder-based, for example liquid polymers and powder particles including polymers and metals. These materials contains some disadvantages such as high melting point and high cost of raw materials [2]. To solve those disadvantages, paste materials have been applied to fabricate three-dimension parts in additive manufacturing. Pastes material is a kind of material composed of solid and liquid state [3]. Pastes are states that are made up from many different substances and formed by using extrusion method. The paste extrusions have been applied for various industries such as ceramic parts, cosmetic pencils, tiles, food manufacturing, animal feeds, and PTFE wires [4]. Extrusion is carried out by pressing pressure to ram extruder, as illustrated in Figure 1 (a). Normally, the ram extrusion consists of control system and extrusion device. In paste extrusion process, the pressure is generated to force the paste through a nozzle. The paste flow is regulated by controlling the ram movement, which moves down to extrude paste material. The extrudate is deposited layer-by-layer on the table to form designed shape. To improve a capability of extrusion process, Extrusion On Demand (EOD), that refer to the ability to regulate the start and stop of paste extrusion, has be developed to improve material deposition. This paper presents a review of process parameters that effect to extrusion on demand such as ram velocity, Dwell time, paste property and extrusion mechanisms.

\section{The paste extrusion process}

The Process of paste extrusion is generally carried out in three steps: paste preparation, Forming, and Finishing [3]. In paste preparation, powder components and liquid are mixed in suitable mass proportion to carry out a paste. These components are contained in the barrel. In forming, Pressure is generated to force the paste through a nozzle. Then, the paste is extruded from an extruder by using ram extrusion. The extrudate is deposited layer-by-layer to form a model. During finishing stage, the extrudate is solidified by thermal processing during this stage. Since a conventional extrusion method extrudes the paste by using ram extruder, the extrudate contained head and tail effect as shown in Figure 1 (b). The quality of designed shape depends on these effects. To achieve the accuracy and quality of designed shape, the conventional extrusion method has been improved to control the start and stop extrusion on demand in order to enhance the performance of the extrusion process. In additional, the ram velocity, Dwell time, paste property and extrusion mechanisms are main parameters that influence on the paste extrusion process. 


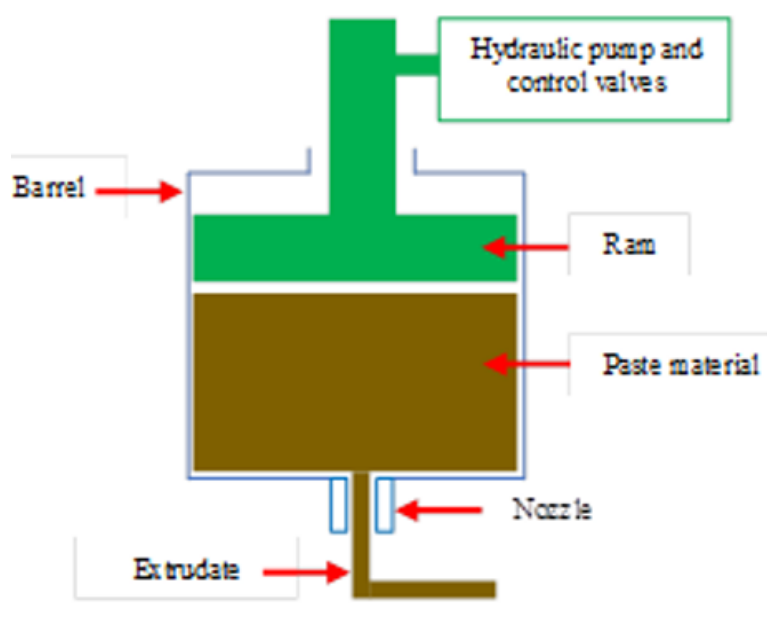

(a) Ram extruder

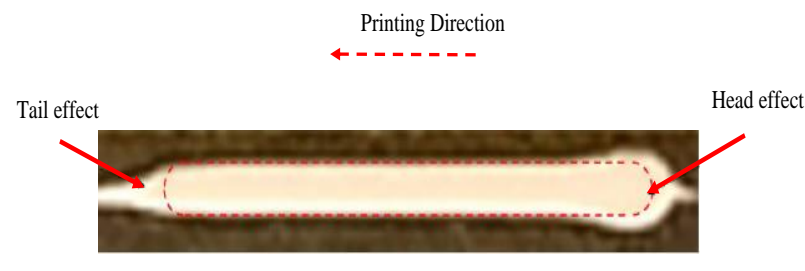

(b) Head and Tail effect [5]

Figure1. Schematic diagram of paste extrusion for Ram extrusion

\subsection{Ram velocity}

The paste can flow when the pressure is high enough to force the paste through the nozzle. The pressure force is generated by the ram extruder. The ram is driven by a hydraulic or mechanical system. Because of the relation between ram velocity and pressure force, it is important that the pressure force is generated sufficiently.

\subsection{Dwell time}

Since there is a delay at start and stop of extrusion, Dwell time is amount of time for start and stop extrusion that process need to compensate the gantry that remains stationary after the force is activated. Determining The Dwell time is important because the excessive Dwell time lead to accumulation of paste material at the start point of printing line. While a few Dwell time cause the nozzle to move forward before the extrusion of paste.

\subsection{Paste properties}

The paste materials were made up from both liquid and solid components, the paste is materials with both a liquid and a solid phase. Paste properties, especially the liquid content and paste viscosity are significant parameter that influences on extrusion process. Variation of liquid contained in paste material cause liquid phase migration that the liquid phase moves faster than the solid particle during extrusion process. So the paste regions of low liquid become drier [6].

\subsection{Extrusion mechanism}

The ram extruder is widely used for conventional paste extrusion. According to a schematic diagram of ram extrusion as shown in Figure 2 (a), which consists of a ram extruder, barrel and nozzle. The paste material is pressed through a nozzle to generate the deposition process. The start and stop of extrusion is regulated by the ram movement. This conventional extrusion is difficult to fabricate complex shape because of the difficultly of determining time delay for the start and stop of extrusion. So, other mechanisms, used for dispensing technology, has been introduced to improve the extrusion on demand. Needle valve extrusion mechanism consists of a plunger, barrel, a shutter needle and nozzle as shown in Figure 2 (b). The shutter needle was set as a function of the opening and closing valve. Paste material was pressed by the force applied to plunger. Then, the shutter needle was moved up or down by pneumatic force to start and stop of extrusion process. Other, auger valve extrusion mechanism consists of servo motor, barrel and auger valve as shown in Figure 2 (c). This valve was used to extrude paste material by the rotating auger valve. The needle and auger valve have been widely used in dispensing of paste materials [7].

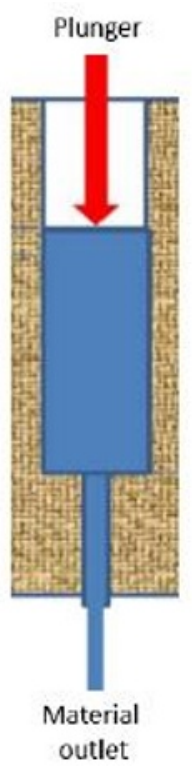

(a) Ram extruder 


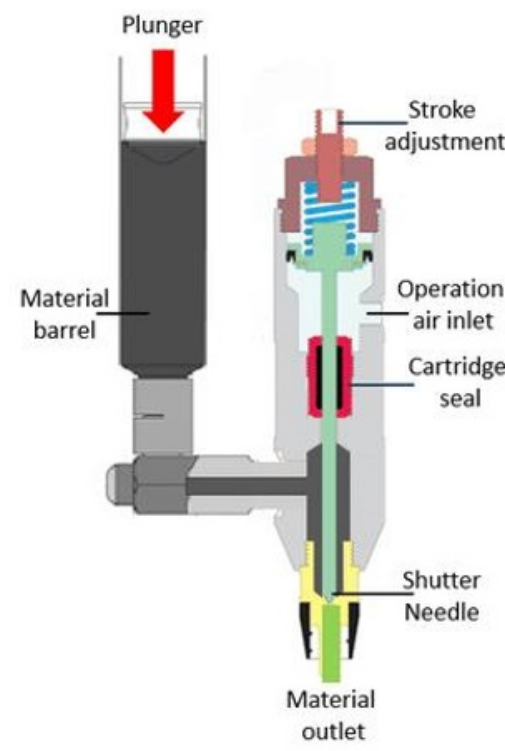

(b) Needle valve

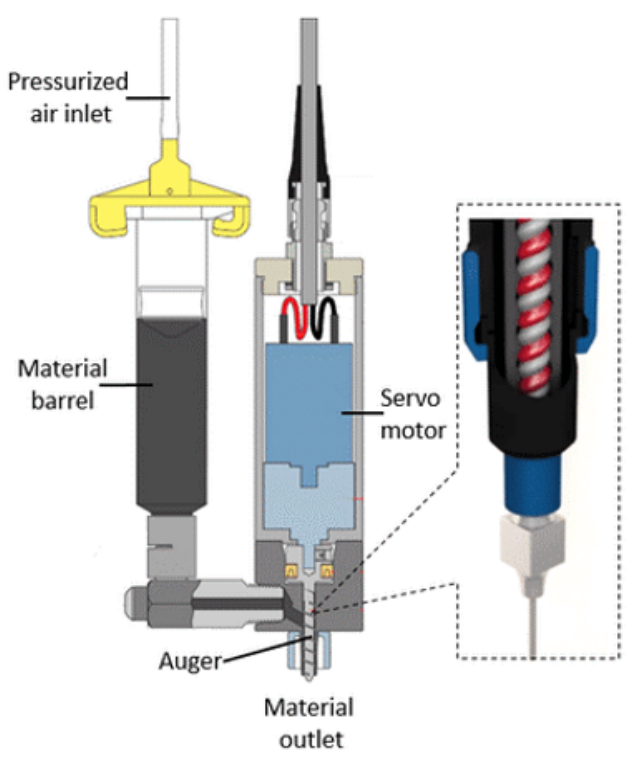

(c) Auger valve

Figure 2. Schematic diagram of ram extruder, needle valve and auger valve extrusion [5]

To improve a capability of extrusion performance, the ram velocity, Dwell time, paste property and extrusion mechanisms are main parameters that have been studies to investigate the effect on the ability extrusion on demand for paste extrusion processes as shown in table 1. Mason et al. (2007) modified the traditional ram extrusion mechanism in order to improve the extrusion on demand. Load cell housing was added to connect directly with the plunger, while plastic syringe was replaced by metal barrel. A new ram extrusion mechanism was implemented to print the line tests with a set of ram retreating velocities $(0,0.25,0.5,1,1.5,2,2.5$, and $3 \mathrm{~mm} / \mathrm{s}$ ) and extrusion forces of $308 \mathrm{~N}$. The results were found that the ram retreating velocities that are higher $2 \mathrm{~mm} / \mathrm{s}$ able to stop on the extrusion on demand. The influence of the ram velocity on the extrusion force and liquid phase migration in Freeze from Extrusion Fabrication process was studied. Liu and Leu (2009) experimented to investigate liquid phase migration for aqueous $\mathrm{Al}_{2} \mathrm{O}_{3}$ paste. The aqueous paste and five ram velocities $(10,5,2,1.5,1 \mu \mathrm{m} / \mathrm{s})$ were used for investigation in this study. The experimental results were shown that highly liquid phase migration was found when ram velocity was lower than $5 \mu \mathrm{m} / \mathrm{s}$. Oake et al. (2009) present an experiment to investigate the Dwell times that effect to extrusion filament. In this experiment, the Dwell time is set as a function of the reference ram force. This force is applied to activate for paste extrusion. Values experiment are $50 \%, 55 \%, 60 \%, 65 \%$ and $70 \%$ of the reference ram force $450 \mathrm{~N}$ and $475 \mathrm{~N}$. The results were shown that the discontinued filaments were presented at Dwell time less than $65 \%$. In addition, the increasing of the reference ram force from 450 to $475 \mathrm{~N}$ was found the accumulation material at the start of filaments. This indicates that a row ram force should be used for extrusion process. Liu et al. (2013) conducted an experiment to study the influence of ram velocity and paste properties on extrusion process by applying series of ram velocities $(2,5,10$ and $15 \mu \mathrm{m} / \mathrm{s})$ and three pastes with difference viscosity. The results were found that the extrusion pressure shown an increasing slowly with high velocities (10 and $15 \mu \mathrm{m} / \mathrm{s})$ whereas the extrusion pressure shown an increasing rapidly at low velocity ( 2 and $5 \mu \mathrm{m} / \mathrm{s}$ ). Therefore, at low velocity, liquid phase was moved into an extrudate. So the remaining paste in the barrel become drier. Drier paste in the barrel need high pressure force to press the paste flow. In addition, the ram velocity of $2 \mu \mathrm{m} / \mathrm{s}$ was experimented to study effect of paste viscosity on liquid phase migration. The results were found that liquid phase migration was found when a higher viscosity paste is used with the lower ram velocity. The paste viscosity had significance effect on extrusion process [12]. The high viscosity of paste materials means a high amount of solid particles. Therefore, high extrusion force had be needed to extrude materials whereas low viscosity paste lead to difficult to form the designed shape. Li et al. (2017) have conducted an experiment to study the influence of extrusion mechanisms, Dwell time and paste property on the start and stop of extrusion. The start Dwell time for ram extrusion, needle valve and auger valve mechanism was 450, 70 and $0 \mathrm{~ms}$ respectively. Those mechanisms were used to experiment the ability of extrusion on demand. Dash line printing tests were conducted for all three methods. The line printing tests were printed from right to left and compared the tail and head effect of the printed lines to indicate the capable extrusion start and stop by using the image dash line segments printed. The experimental results were shown that the dash line segments printed by the needle valve and auger valve methods have shorter tails than the ram extrusion method because the start and stop dwell time of the needle valve and auger valve methods are shorter than the ram extrusion method. Then, the filaments with short start Dwell time shown the accuracy of the start and stop of extrusion. 
Table 1. Overview of the main extrusion process parameters that effect to extrusion on demand

\begin{tabular}{|c|c|c|c|c|}
\hline References & Ram velocity & Dwell time & Past property & Extrusion mechanism \\
\hline$[8]$ & $\checkmark$ & - & - & - \\
\hline$[9]$ & $\checkmark$ & - & $\checkmark$ & - \\
\hline$[10]$ & $\checkmark$ & $\checkmark$ & - & - \\
\hline$[11]$ & $\checkmark$ & - & $\checkmark$ & $\checkmark$ \\
\hline$[5]$ & - & $\checkmark$ & $\checkmark$ & \\
\hline
\end{tabular}

\section{Conclusion}

The extrusion methods have been widely used to fabricate $3 \mathrm{D}$ objects from paste materials. Those materials are made up from liquid and solid particle. The ram extrusion method is widely used to form paste materials. In this method, pressure force was generated to press paste material through a nozzle by using ram extruder. To improve a capability of extrusion process. The four main parameters were reviewed. The ram velocity, Dwell time, paste property and extrusion mechanisms are those parameters that influence on the paste extrusion process. The ram extruder, needle valve and auger valve based extrusion method were experimented to print the line tests. Because of the shorter Dwell time, needle valve and auger valve shown the ability start and stop of extrusion on demand better than the ram extrusion.

\section{References}

1. ASTM Standard F2792-12a. (2012). Standard Terminology for Additive Manufacturing Technologies. ASTM International, West Conshohocken, PA.

2. Lee, J.-Y., J. An and C. K. Chua. (2017). Fundamentals and applications of 3D printing for novel materials. Applied Materials Today 7(Supplement C): 120-133.

3. J. Benbow and J. Bridgwater. (1993). Paste flow and extrusion. Oxford series on advanced manufacturing, Oxford.

4. Amza, C., A. Zapciu and D. Popescu (2017). Paste Extruder-Hardware Add-On for Desktop 3D Printers. Technologies 5(3): 50.
5. Li, W., A. Ghazanfari, M. C. Leu and R. G. Landers., (2017). Extrusion-on-demand methods for high solids loading ceramic paste in freeform extrusion fabrication.Virtual and Physical Prototyping 12(3): 193-205

6. Rough, S. L., J. Bridgwater and D. I. Wilson., (2000). Effects of liquid phase migration on extrusion of microcrystalline cellulose pastes. International Journal of Pharmaceutics 204(1): 117-126.

7. Jianping, L. and G. Deng., (2004). Technology development and basic theory study of fluid dispensing - a review.

8. S. Mason, M., T. Huang, R. G. Landers, M. Leu, G. Hilmas and M. W. Hayes., (2007). Aqueous-Based Extrusion Fabrication of Ceramics on Demand.

9. Liu, H. and M. C. LEU., (2009). Liquid Phase Migration In Extrusion Of Aqueous Alumina Paste For Freeze-Form Extrusion Fabrication. International Journal of Modern Physics B 23(06n07): 1861-1866.

10. Oakes, T., P. Kulkarni, R. G Landers and M. Leu., (2009). Development of extrusion-on-demand for ceramic Freeze-form Extrusion Fabrication.

11. Liu, H., J. Liu, M. C. Leu, R. Landers and T. Huang (2013). Factors influencing paste extrusion pressure and liquid content of extrudate in freeze-form extrusion fabrication. The International Journal of Advanced Manufacturing Technology 67(1): 899906.

12. Hong, S., C. Sanchez, H. Du and N. Kim., (2015). Fabrication of 3D Printed Metal Structures by Use of High-Viscosity $\mathrm{Cu}$ Paste and a Screw Extruder. Journal of Electronic Materials 44(3): 836-841. 